\title{
Thromboembolic events, bleeding, and drug discontinuation in patients with atrial fibrillation on anticoagulation: a prospective hospital-based registry
}

\author{
Oliver Königsbrügge ${ }^{1 *}$ (D) Alexander Simon², Hans Domanovits², Ingrid Pabinger ${ }^{1}$ and Cihan Ay ${ }^{1,3}$
}

\begin{abstract}
Background: The clinical practice of stroke prevention in atrial fibrillation (AF) with direct oral anticoagulants (DOACS) differs from anticoagulation in randomized trial patients. We investigated the risk of thromboembolism, bleeding, and drug discontinuation in a hospital-based real-world setting.

Methods: All-comer patients with non-valvular AF were recruited into a registry at an academic tertiary care center. After informed consent, patients underwent a personal structured interview including medical history, past and current anticoagulation, and returned for follow-up after 6-12 months.

Results: The registry comprised 282 patients (42\% women, median age 71 years) with a median CHA2DS2-Vasc-Score of 4 (25. to 75. percentile 2.5-5), who were prospectively followed 285 days in median. At inclusion, 118 patients took vitamin-K-antagonists, 33 dabigatran, 87 rivaroxaban, 30 apixaban, 5 low-molecular-weight heparin, and 9 were on no anticoagulant. Occurrence of stroke (rate 2.8/100 patient-years), was associated with prior stroke (hazard ratio [HR] 18.5, 95\% confidence interval 2.16-159), increased HbA1c (HR per 1\% increase 1.71, 1.20-2.45) and borderline significantly associated with vascular disease (HR 8.33, 0.97-71.3). Further we observed a high rate of major bleeding (2.8/100 patient-years), clinically relevant non-major bleeding (4.1/100 patient-years), and venous thromboembolism (2.8/100 patient-years). Anticoagulation was discontinued by 80 patients (36.9/100 patient-years), and diabetes (HR 2.31, 1.32-4. 02), history of bleeding (HR 2.51, 1.44-4.37) and elevated leucocyte count (HR per 1G/I increase 1.02, 1.00-1.05) were associated with increased risk of discontinuation.
\end{abstract}

Conclusions: In this hospital-based registry, patients with atrial fibrillation had an increased risk of thromboembolic events despite anticoagulation. The low drug persistence may be attributable to distinct comorbid conditions and bleeding complications.

Keywords: Atrial fibrillation, Anticoagulation, Tertiary healthcare, Stroke, Hemorrhage, Medication persistence

\section{Background}

The increased risk of stroke and systemic embolism in patients with non-valvular atrial fibrillation (AF) can be attenuated with continuous oral anticoagulation treatment [1]. Direct oral anticoagulants (DOACs), including the direct thrombin inhibitor dabigatran and the direct factor

\footnotetext{
* Correspondence: oliver.koenigsbruegge@meduniwien.ac.at 'Department of Medicine I, Clinical Division of Hematology \& Hemostaseology, Medical University of Vienna, Währinger Gürtel 18-20, A-1090 Vienna, Austria

Full list of author information is available at the end of the article
}

Xa inhibitors rivaroxaban, apixaban, and edoxaban, have shifted the paradigm of anticoagulation treatment from routine drug monitoring and dose adjustment to a onesize-fits-all strategy, and changed clinical practice of oral anticoagulation. Real-world data have confirmed the efficacy and safety of DOACs for stroke prevention $[2,3]$. However, in a hospital-based patient population with AF, comorbid conditions, comedications and surgical interventions may complicate treatment with anticoagulant drugs, which has not been specifically addressed in previous realworld investigation. 
Furthermore, there is still concern that the ease of drug administration and clinical management with DOACs may not guarantee better persistence on treatment in a real-world setting. Drug persistence is defined as the total time a patient stays on a prescribed medication and reduced persistence increases the risk of stroke $[4,5]$. In the randomized controlled trials that led to the licensing of DOACs for stroke prevention in AF, 20.7\%-34.3\% of patients receiving DOACs discontinued drug treatment during the study period and $16.6 \%-34.4 \%$ discontinued the control treatment, warfarin [6-9]. However, drug persistence in clinical trials may be completely different from real-world persistence, because there are different incentives for remaining on treatment in real-world patients.

We aimed to examine the characteristics of non-valvular AF patients in a hospital-based setting and investigate risk factors for occurrence of thromboembolic events, bleeding episodes and drug persistence while on anticoagulant treatment, as well as reasons for drug discontinuation.

\section{Methods \\ Patients}

Patients with non-valvular AF referred to the Clinical Division of Hematology and Hemostaseology were recruited into a hospital-based, prospective registry from July 2013 to May 2016. Inclusion criteria were confirmed diagnosis of AF by a 12-channel, resting electrocardiogram (ECG), willingness to comply with study procedures, and written informed consent. The study has approval of the local ethics committee. There were no exclusion or selection criteria concerning anticoagulation treatment, medical history, risk of stroke, or bleeding. After obtaining informed consent for study participation, a study investigator performed a personal structured interview with each patient, recorded the medical history and a detailed anticoagulation history. Patients returned for voluntary, scheduled follow-up visits 6 and 12 months after recruitment.

\section{Baseline assessment of patient characteristics}

All baseline patient data was recorded from personal interviews and verified against medical records. The structured interview included a detailed medical history, especially concerning prior thromboembolic events, and a detailed record of previous periods of anticoagulation treatment and associated complications. During baseline data collection, a history of vascular disease was defined as arterial disease of any degree including coronary heart disease, peripheral artery disease, and carotid artery disease. Cancer was defined as any history of malignancy or active malignancy at study inclusion with the exception of basalioma. Patients were anticoagulation naive at baseline if they had not received continuous anticoagulation treatment previously for longer than 3 months. The baseline kidney function was calculated on the grounds of the estimated glomerular filtration rate (eGFR) and isotope-dilution mass spectrometry (IDMS) traceable serum creatinine levels using the Modification of Diet in Renal Disease (MDRD) equation. The risk of stroke was assessed with the $\mathrm{CHA}_{2} \mathrm{DS}_{2}$-Vasc score and the risk of bleeding with the HAS-BLED score.

\section{Definition of events during prospective observation}

Bleeding outcomes were classified according to the recommendations of the Scientific and Standardization Committee of the International Society of Thrombosis and Hemostasis. Major bleeding was defined as clinically overt bleeding with fatal outcome, involvement of a critical anatomic site, fall in hemoglobin concentration of more than $2 \mathrm{~g} / \mathrm{dl}$, transfusion of $>2$ units of whole blood or packed red blood cells, or leading to permanent disability [10]. Clinically relevant non-major (CRNM) bleeding was defined as overt bleeding not meeting criteria for major bleeding but requiring medical intervention, hospitalization, temporary interruption or delayed dosing of anticoagulation, pain, or impairment of daily activities [11]. Minor bleeding was defined as any other bleeding not meeting the above criteria. Venous thromboembolism (VTE) was defined as objectively confirmed deep vein thrombosis (DVT) or pulmonary embolism (PE). Stroke was defined as the sudden onset of a distinct focal neurologic deficit in a location consistent with the territory of a major cerebral artery and conclusive imaging evidence. In the absence of imaging evidence, but conclusive neurological symptoms confirmed by a specialist in neurology, we recorded a transient ischemic attack (TIA). All events were internally validated according to the criteria listed above.

\section{Definition of anticoagulation persistence}

Drug persistence was defined as the time in weeks from the initiation of anticoagulation until discontinuation of treatment [12]. Only patients who initiated anticoagulation at the time of recruitment were included in the persistence analysis. The percentage of patients remaining on the baseline anticoagulant was calculated for 6 and 12 months as well as the annualized discontinuationrate. The end of anticoagulation persistence was defined as the permanent cessation of anticoagulation treatment or switching from one anticoagulant to a different anticoagulant, but did not include pausing anticoagulation treatment for a limited time of less than 1 month.

\section{Statistical methods}

Baseline patient characteristics of the registry cohort were described as absolute and relative frequencies or with median and $25^{\text {th }}$ to $75^{\text {th }}$ percentile, respectively for categorical and continuous data. The baseline group comparisons between DOAC and vitamin-K-antagonist 
(VKA) patients were calculated with the Mann-Whitney$\mathrm{U}$ test for categorical parameters or the $\mathrm{chi}^{2}$ test for continuous variables. An asymptotic two-sided $p$-value of smaller than 0.05 was considered statistically significant. The risks of stroke, TIA, or systemic embolism, bleeding, and drug discontinuation were calculated with the univariable Cox proportional hazards model. All calculations were performed with SPSS (Windows Version 23.0; IBM Corp., Armonk, NY, http://www.ibm.com). Risk of discontinuation was only calculated with patients who initiated a new anticoagulation therapy at study inclusion.

\section{Results}

\section{Baseline registry characteristics}

The registry includes 282 patients (118 [42\%] women, 164 [58\%] men) with ECG-confirmed diagnosis of AF and a median age of 71 years $\left(25^{\text {th }}\right.$ to $75^{\text {th }}$ percentile 65 - 77 years). At baseline, 118 patients $(41.8 \%)$ were on VKA, 33 patients (11.7\%) on dabigatran, 87 (30.9\%) on rivaroxaban, $30(10.6 \%)$ on apixaban, 5 patients $(1.8 \%)$ on long-term low-molecular-weight heparin (LMWH) and 9 patients $(3.2 \%)$ did not take any anticoagulant drug. The baseline characteristics of the full registry and respective to groups of patients on each anticoagulant are shown in Table 1. DOACs were more frequently prescribed to patients with more recently diagnosed AF and to patients previously naïve to anticoagulation treatment (Table 1). Patients with history of CRNM or major bleeding, with age between 65-75 years were also more frequently on DOAC treatment. Patients older than 75 years were more often on VKA, while patients on DOACs, especially rivaroxaban and apixaban, tended to have lower hemoglobin and hematocrit levels than patients on VKA. Patients with lower eGFR, however, tended to take VKA (Table 1).

\section{Prospective outcomes}

Prospective follow-up was available for 269 patients and 13 patients (4.6\%) were lost to follow-up. The median observation time was 285 days (227-405 days) (minimum 1 day, maximum 966) for a total of 217 patient-years of observation time. During follow-up, 6 (2.2\%) cardioembolic events occurred (4 ischemic strokes, 1 TIA, 1 systemic embolism), corresponding to an event-rate of 2.8 per 100 patient-years. Of these events, 4 occurred while on VKA, 1 while on rivaroxaban, and 1 while on triple therapy with VKA.

In univariable Cox regression, patients with a history of stroke, TIA, or systemic embolism had an 18-fold increased risk of a new stroke, TIA or systemic embolism (hazard ratio [HR] 18.5, 95\% confidence interval [CI] $2.16-159, p=0.008$ ), patients with vascular disease had a borderline significant 8.3-fold increased risk of stroke (HR 8.3, 95\% CI $0.97-71.3, p=0.05$ ), and for every $1 \%$ increase in $\mathrm{HbA} 1 \mathrm{c}$ the risk of stroke increased 1.7-fold (95\% CI 1.20-2.45, $p=0.003$ ) (Table 2). For every one point added to the CHA2DS2-Vasc score the risk of stroke doubled (HR 2.06, 95\% CI 1.27-3.35, $p=0.004$ ). One myocardial infarction occurred while on VKA, and 6 VTE events occurred (4 DVT, $2 \mathrm{PE}$ ), corresponding to an event-rate of 2.8 per 100 patient-years. One VTE event occurred while on rivaroxaban therapy, 2 during rivaroxaban pause, 1 during VKA treatment, 1 during VKA pause, and 1 after discontinuation of rivaroxaban. Major bleeding events occurred in 6 patients ( 3 intraocular hemorrhages, 2 intraabdominal bleedings with massive blood loss and/or transfusions, 1 subdural hematoma) and CRNM bleeding events occurred in 9 patients (5 gastrointestinal [GI] bleeds, 4 other bleeds requiring hospitalization). The event rate for major bleeding was 2.8 per 100 patient-years and for CRNM bleeding 4.1 per 100 patient-years. Minor bleeding events occurred in 43 patients and 15 patients died during follow-up of causes unrelated to atrial fibrillation or anticoagulation. The results of our analysis revealed no statistically significant risk factor for bleeding that occurred during follow-up.

\section{Anticoagulation persistence}

The median persistence was 32 weeks $\left(25^{\text {th }}\right.$ to $75^{\text {th }}$ percentile: 12 to 46 weeks) and 80 patients (29.7\%) discontinued the anticoagulation therapy, which they had received at baseline. This corresponds to a rate of discontinuation of 36.9 per 100 patient-years. After 6 months, the overall drug persistence of the baseline anticoagulant was $76.7 \%$ and after 12 months further reduced to $54.7 \%$. There was no difference in the persistence between patients receiving DOACs and VKA (Fig. 1). The most frequent reasons for discontinuing anticoagulation were patient-reported end of AF and permanent return to sinus rhythm (20\%), emergence of a new contraindication for current anticoagulation treatment (15\%) (e.g. mechanical heart valve), physician's recommendation (12.5\%), and occurrence of major or CRNM bleeding events (11.3\%) (Table 3). The choice for an alternative anticoagulant after discontinuation of the baseline anticoagulant drug was evenly distributed between VKA, rivaroxaban, apixaban or no anticoagulant at all (Table 3). In regression analysis, patients with diabetes had a 2.3 -fold increased risk of discontinuation (95\% CI 1.32 to 4.02), patients with history of bleeding had a 2.5 -fold increased risk (95\% CI 1.44 to 4.37) and per $1 \mathrm{G} / \mathrm{l}$ increase in leucocyte count the risk of discontinuation increased by $2 \%(\mathrm{HR}=1.02,95 \%$ CI 1.00 to 1.05$)$.

\section{Discussion}

In this analysis of a real-world, tertiary-care, hospitalbased registry of patients with atrial fibrillation, the rate 
Table 1 Baseline cohort characteristics

\begin{tabular}{|c|c|c|c|c|c|c|c|c|}
\hline \multirow[t]{2}{*}{ Characteristic } & \multirow{2}{*}{$\begin{array}{l}\text { Full cohort } \\
(N=282)\end{array}$} & \multirow[t]{2}{*}{$\operatorname{VKA}(N=118)$} & \multicolumn{2}{|c|}{ Dabigatran $(N=33)$} & \multicolumn{2}{|c|}{ Rivaroxaban $(N=87)$} & \multicolumn{2}{|l|}{ Apixaban $(N=30)$} \\
\hline & & & & $p^{*}$ & & $p^{*}$ & & $p^{*}$ \\
\hline Age & $71(65-77)$ & $73(67-79)$ & $73(68-77)$ & 0.8 & $71(66-75)$ & 0.1 & $71(64-75)$ & 0.04 \\
\hline Female sex & $116(43.1)$ & 47 (41.6) & $11(34.4)$ & 0.5 & $30(37.0)$ & 0.5 & $22(75.9)$ & 0.001 \\
\hline BMl & $27.0(24.5-30.4)$ & $27.3(24.9-31.2)$ & $27.3(25.0-31.0)$ & 0.7 & $27.0(24.0-31.1)$ & 0.5 & $26.9(22.2-28.3)$ & 0.09 \\
\hline \multicolumn{9}{|l|}{ Type of AF } \\
\hline Recently diagnosed & $52(18.4)$ & $10(8.5)$ & $8(24.2)$ & 0.04 & $16(18.4)$ & 0.2 & $13(43.3)$ & $<0.001$ \\
\hline paroxysmal & $131(46.5)$ & $60(50.8)$ & $10(30.3)$ & 0.05 & $42(49.4)$ & 0.8 & $12(40.0)$ & 0.3 \\
\hline persisting & $19(6.7)$ & $9(7.6)$ & $1(3.0)$ & 0.4 & $7(8.6)$ & 0.7 & $1(3.3)$ & 0.5 \\
\hline permanent & $80(28.4)$ & $39(33.1)$ & $14(42.4)$ & 0.3 & $22(27.2)$ & 0.4 & $4(13.3)$ & 0.04 \\
\hline Time since AF diagnosis, years & $4(1-8)$ & $4(2-9)$ & $5(0.1-9)$ & 0.5 & $3(1-10)$ & 0.1 & $2(0-6)$ & 0.01 \\
\hline History of electrical cardioversion & $83(30.9)$ & $40(35.4)$ & $10(31.3)$ & 0.7 & $21(25.9)$ & 0.2 & $9(31.0)$ & 0.7 \\
\hline History of ablation & $35(13.0)$ & $15(13.3)$ & $5(15.6)$ & 0.7 & $13(16.0)$ & 0.6 & $1(3.4)$ & 0.1 \\
\hline Family history of AF & $33(12.3)$ & $13(11.5)$ & $3(9.4)$ & 0.7 & $10(12.3)$ & 0.9 & $7(24.1)$ & 0.08 \\
\hline \multicolumn{9}{|l|}{ Medical history } \\
\hline Congestive heart failure & $81(30.1)$ & $34(30.1)$ & $13(40.6)$ & 0.3 & $24(29.6)$ & 0.9 & $8(27.6)$ & 0.8 \\
\hline Hypertension & $227(84.4)$ & 99 (87.6) & $30(93.8)$ & 0.3 & $65(80.2)$ & 0.2 & $26(89.7)$ & 0.8 \\
\hline Age $\geq 75$ years & $98(36.4)$ & $53(46.9)$ & $13(40.6)$ & 0.5 & $23(28.4)$ & 0.009 & $8(27.6)$ & 0.06 \\
\hline Age $65-74$ years & $114(42.4)$ & $41(36.3)$ & $14(43.8)$ & 0.4 & $43(53.1)$ & 0.02 & $13(44.8)$ & 0.4 \\
\hline Diabetes & $81(28.7)$ & $31(27.4)$ & $10(31.3)$ & 0.4 & $22(27.2)$ & 0.7 & $8(27.6)$ & 0.9 \\
\hline Stroke / TIA / systemic embolism & $55(20.4)$ & $22(19.5)$ & $7(21.9)$ & 0.8 & $20(24.7)$ & 0.4 & $4(13.8)$ & 0.5 \\
\hline Vascular disease & $96(35.7)$ & $41(36.3)$ & $13(40.6)$ & 0.7 & $28(34.6)$ & 0.8 & $11(37.6)$ & 0.9 \\
\hline $\mathrm{CHA}_{2} \mathrm{DS}_{2}$-VASC Score & $4(2.5-5)$ & $4(3-5)$ & $4(3-5)$ & 0.9 & $3(2-5)$ & 0.3 & $4(3-4.5)$ & 0.4 \\
\hline HAS-BLED Score & $2(1-3)$ & $2(1-2)$ & $2(1-3)$ & 0.7 & $2(1-3)$ & 0.2 & $2(1-3)$ & 0.5 \\
\hline Abnormal liver or kidney function & $52(19.3)$ & $24(21.2)$ & $5(15.6)$ & 0.5 & $18(22.2)$ & 0.9 & $5(17.2)$ & 0.6 \\
\hline CRNM bleeding & $13(4.8)$ & $1(0.9)$ & $2(6.3)$ & 0.06 & $4(4.9)$ & 0.08 & $4(13.8)$ & 0.001 \\
\hline Major bleeding & $10(3.7)$ & $1(0.9)$ & $5(15.6)$ & $<0.001$ & $4(4.9)$ & 0.08 & 0 & 0.6 \\
\hline Venous thromboembolism & $22(8.2)$ & $7(6.2)$ & $3(9.4)$ & 0.5 & $9(11.1)$ & 0.2 & $3(10.3)$ & 0.4 \\
\hline Cancer & $58(21.6)$ & 19 (16.8) & $9(28.1)$ & 0.2 & $19(23.5)$ & 0.3 & $6(20.7)$ & 0.6 \\
\hline OAC-naïve at baseline & $18(6.7)$ & $4(3.5)$ & $2(6.3)$ & 0.5 & $6(7.4)$ & 0.2 & $4(13.8)$ & 0.03 \\
\hline Aspirin comedication & $58(21.6)$ & $23(20.4)$ & $5(15.6)$ & 0.6 & $19(23.5)$ & 0.6 & $6(20.7)$ & 0.9 \\
\hline Clopidogrel comedication & $14(5.2)$ & $9(8.0)$ & $2(6.3)$ & 0.7 & $3(3.7)$ & 0.2 & 0 & 0.1 \\
\hline \multicolumn{9}{|l|}{ Baseline laboratory parameters } \\
\hline Platelet count & $214(176-264)$ & $211(180-251)$ & $203(172-239)$ & 0.4 & $215(174-286)$ & 0.3 & $238(177-275)$ & 0.4 \\
\hline Hemoglobin & $13.2(11.8-14.4)$ & $13.5(12.5-14.8)$ & $13.2(11.8-15.2)$ & 0.3 & $12.7(11.4-14.1)$ & 0.002 & $12.8(11.0-14.1)$ & 0.05 \\
\hline Hematocrit & $39.5(35.1-42.8)$ & $40.3(37.4-43.9)$ & $39.5(34.9-42.6)$ & 0.2 & $38.5(33.6-41.0)$ & 0.001 & $38.8(33.9-41.2)$ & 0.03 \\
\hline Leucocyte count & $6.8(5.7-8.5)$ & $6.8(5.7-7.9)$ & $7.0(6.0-8.6)$ & 0.3 & $6.9(5.7-8.9)$ & 0.3 & $6.4(5.2-7.9)$ & 0.3 \\
\hline $\operatorname{eGFR}\left(\mathrm{ml} / \mathrm{min} / 1.73 \mathrm{~m}^{2}\right)$ & $65.2(53.3-79.6)$ & $60.4(49.5-74.3)$ & $71.2(58.4-91.8)$ & 0.03 & $66.8(56.2-82.1)$ & 0.01 & $65.8(56.6-73.1)$ & 0.2 \\
\hline
\end{tabular}

Footnote: * Mann-Whitney-U test or $\mathrm{chi}^{2} p$-value for asymptomatic two-sided difference between respective DOAC group and VKA group, statistically significant $p$-values in bold print

Abbreviations: $A F$ atrial fibrillation, VKA Vitamin-K-antagonist, BMI body-mass-index, TIA transient ischemic attack, CRNM bleeding clinically relevant non-major bleeding, $O A C$ oral anticoagulation, $e G F R$ estimated glomerular filtration rate

of cardioembolic events (2.8 per 100 patient-years) was higher than in the randomized controlled trials for stroke prevention in AF. In RE-LY, ROCKET-AF, ARISTOTLE and ENGAGE-AF-TIMI48, 1.6-2.2\% of patients randomized to warfarin and $1.11-2.04 \%$ for patients randomized to the study drugs had strokes or systemic embolisms per year [6-9]. The stroke-rate in our study was also higher than in other data sets of real-world AF 
Table 2 Univariable Cox regression analysis of risk factors for the outcomes stroke, bleeding, and drug discontinuation

\begin{tabular}{|c|c|c|c|c|c|c|}
\hline \multirow[b]{2}{*}{ Characteristic } & \multicolumn{2}{|c|}{$\begin{array}{l}\text { Hazard of stroke, TIA, systemic } \\
\text { embolism }(N=269)\end{array}$} & \multicolumn{2}{|c|}{$\begin{array}{l}\text { Hazard of CRNM or major bleed } \\
(N=269)\end{array}$} & \multicolumn{2}{|l|}{$\begin{array}{l}\text { Hazard of anticoagulant } \\
\text { discontinuation }(N=144)\end{array}$} \\
\hline & $\begin{array}{l}\text { Hazard ratio } \\
\text { (95\% confidence interval) }\end{array}$ & $p$-value & $\begin{array}{l}\text { Hazard ratio } \\
\text { ( } 95 \% \text { confidence interval) }\end{array}$ & $p$-value & $\begin{array}{l}\text { Hazard ratio } \\
\text { ( } 95 \% \text { confidence interval) }\end{array}$ & $p$-value \\
\hline$\overline{\text { Age }^{a}}$ & $0.98(0.90-1.06)$ & 0.59 & $1.03(0.97-1.09)$ & 0.30 & $0.99(0.96-1.02)$ & 0.54 \\
\hline Female sex & $6.45(0.75-55.2)$ & 0.09 & $0.95(0.36-2.56)$ & 0.93 & $1.01(0.59-1.75)$ & 0.96 \\
\hline $\mathrm{BMI}^{\mathrm{a}}$ & $0.96(0.81-1.13)$ & 0.63 & $1.00(0.92-1.10)$ & 0.96 & $0.99(0.94-1.04)$ & 0.65 \\
\hline \multicolumn{7}{|l|}{ Medical history } \\
\hline Congestive heart failure & $2.00(0.40-9.94)$ & 0.40 & $1.15(0.42-3.18)$ & 0.78 & $1.28(0.73-2.23)$ & 0.39 \\
\hline Hypertension & $24.2(0.00-999)$ & 0.60 & $23.8(0.01-999)$ & 0.42 & $0.60(0.28-1.28)$ & 0.18 \\
\hline Diabetes & $2.42(0.49-12.0)$ & 0.28 & $1.13(0.39-3.27)$ & 0.82 & $2.31(1.32-4.02)$ & 0.003 \\
\hline Stroke/TIA/systemic embolism & $18.5(2.16-159)$ & 0.008 & $0.76(0.22-2.69)$ & 0.67 & $0.76(0.38-1.53)$ & 0.44 \\
\hline Vascular disease & $8.33(0.97-71.3)$ & 0.05 & $0.62(0.21-1.82)$ & 0.39 & $1.16(0.67-2.00)$ & 0.61 \\
\hline $\mathrm{CHA}_{2} \mathrm{DS}_{2}-$ VASC Score ${ }^{\mathrm{a}}$ & $2.06(1.27-3.35)$ & 0.004 & $1.03(0.77-1.37)$ & 0.85 & $1.05(0.90-1.21)$ & 0.57 \\
\hline History of bleeding & $0.85(0.10-7.27)$ & 0.88 & $1.17(0.33-4.15)$ & 0.81 & $2.51(1.44-4.37)$ & 0.001 \\
\hline HAS-BLED Score ${ }^{a}$ & $1.61(0.90-2.87)$ & 0.11 & $1.18(0.77-1.80)$ & 0.45 & $1.14(0.92-1.42)$ & 0.22 \\
\hline Venous thromboembolism & $2.26(0.26-19.4)$ & 0.46 & $0.87(0.11-6.64)$ & 0.89 & $1.29(0.51-3.26)$ & 0.59 \\
\hline Active cancer/history of cancer & $0.04(0.00-330)$ & 0.48 & $1.24(0.35-4.40)$ & 0.74 & $1.15(0.62-2.12)$ & 0.66 \\
\hline OAC-naïve at baseline & $0.05(0.00-999)$ & 0.69 & $0.94(0.12-7.23)$ & 0.95 & $0.78(0.31-1.97)$ & 0.60 \\
\hline \multicolumn{7}{|c|}{ Baseline laboratory parameters (hazard ratios per 1 unit increase) } \\
\hline Platelet count ${ }^{a}$ & $1.00(0.99-1.01)$ & 0.71 & $1.00(0.99-1.01)$ & 0.60 & $1.00(0.99-1.00)$ & 0.34 \\
\hline Hemoglobin ${ }^{a}$ & $0.80(0.52-1.22)$ & 0.29 & $1.05(0.81-1.37)$ & 0.71 & $0.89(0.79-1.02)$ & 0.08 \\
\hline Hematocrit $^{a}$ & $0.95(0.81-1.11)$ & 0.51 & $1.01(0.91-1.11)$ & 0.86 & $0.96(0.92-1.01)$ & 0.11 \\
\hline Leucocyte count $^{\mathrm{a}}$ & $1.02(0.91-1.13)$ & 0.77 & $0.82(0.63-1.06)$ & 0.12 & $1.02(1.00-1.05)$ & 0.041 \\
\hline eGFR ${ }^{a}$ & $0.97(0.93-1.01)$ & 0.17 & $0.98(0.96-1.01)$ & 0.21 & $1.01(1.00-1.02)$ & 0.27 \\
\hline $\mathrm{HbA} 1 c^{a}$ & $1.71(1.20-2.45)$ & 0.003 & $1.13(0.75-1.72)$ & 0.54 & $1.31(0.87-1.98)$ & 0.20 \\
\hline
\end{tabular}

Legend: BMI body-mass index, TIA transient ischemic attack, VKA vitamin-K-antagonist, OAC oral anticoagulant, eGFR estimated glomerular filtration rate, 999 as the upper bound of the $95 \%$ confidence interval signifies an abbreviation of a very wide confidence interval, $p$-values in bold font represent statistically significant findings, ${ }^{a}$ the hazard ratios for continuous variables are given as per 1 unit increase: age in years, BMI in $\mathrm{kg} / \mathrm{m}^{2}$, platelet count in $\mathrm{G} / \mathrm{l}$, hemoglobin in $\mathrm{g} / \mathrm{dl}$, hematocrit in \%, leucocyte count in G/l, eGFR in $\mathrm{ml} / \mathrm{min} / 1.73 \mathrm{~m}^{2}, \mathrm{HbA} 1 \mathrm{c}$ in rel.\%, and D-dimer in $\mu \mathrm{g} / \mathrm{ml}$

patients. Graham et al. reported a stroke rate of 1.1 per 100 patient years for dabigatran and 1.4 for warfarin in a database of US Medicare patients [13]. Korenstra et al. found a rate of stroke of $0.8 \%$ per year for VKA and $1.0 \%$ per year for dabigatran outpatients [14], and Hecker et al. reported an event rate of 2.0 per 100 patient-years in rivaroxaban patients in daily-care [15]. In a recent report from the GARFIELD-AF registry, the rate of stroke was 1.25 per 100 patient-years [16]. Not surprisingly, these cohorts had lower frequencies of risk factors for strokes, including a prior history of stroke, a history of vascular disease, diabetes and a decreased CHA2DS2-Vasc Score, compared to our cohort. This is confirmation that a stroke risk evaluation is clinically meaningful in a hospital-based setting. We also observed a rate of myocardial infarction of 0.5 per 100 patients-years and a rate of VTE of 2.8 per 100 patientyears. Firstly, it is interesting that a cohort reported by Beyer-Westendorf et al. from a population of primary-care AF patients with similar baseline stroke risk factors and similar stroke rate had no VTE events at all [2]. Thus, the risk of VTE in AF patients, especially when medically ill or frail, should not be neglected. Secondly, a closer inspection of the circumstances under which VTE events occurred, revealed that the majority occurred during temporary pausing of the anticoagulation therapy. Thus, VTE events may have been avoidable with a proper bridging strategy or minimizing of the anticoagulation pause. We would like to interpret this signal as a call to action on emphasizing educational programs on safe and efficacious anticoagulation management during interventions, operations, and hospitalizations.

Major bleeding events occurred with a rate of 2.8 per 100 patient-years and CRNM bleeding events with a rate of 4.1 per 100 patient-years. The rate of major or CRNM bleeding events was, however, not elevated in our cohort compared to some other real-world registries despite a median age of 71 years and a history of severe bleeding complications in $8.5 \%$ of patients. This may be an indication that bleeding risk evaluation in medically ill 


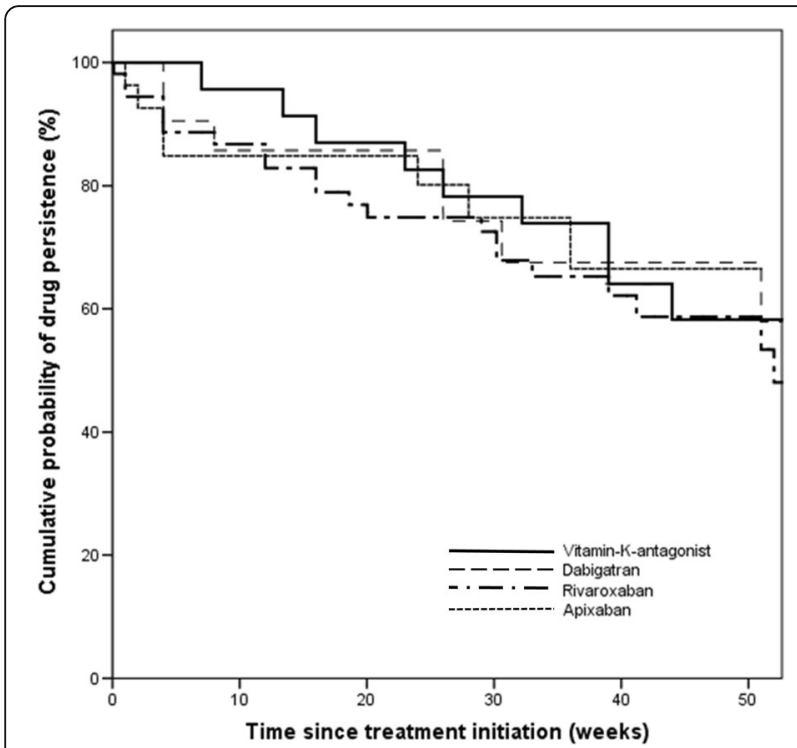

Fig. 1 Cumulative drug persistence over the course of 50 weeks

patients may be different from the general population and that bleeding assessment tools, such as the HASBLED score, should be updated to the DOAC era. Three patients, without diabetes or previous history of intraocular or retinal bleeding, suffered from symptomatic, intraocular hemorrhage during follow-up. Bleeding in this atypical site has previously been reported in trials on anticoagulation in AF patients [7], but the high

Table 3 Reasons for discontinuation of first choice anticoagulant and frequency of alternative choice anticoagulants $(\mathrm{N}=80)$

Frequency (\%)

Reason for discontinuation

Patient-reported permanent return to sinus rhythm

$16(20.0)$

Contraindication

$12(15.0)$

Physician's recommendation

$10(12.5)$

Difficulty reaching INR 2-3 (VKA only)

$9(11.3)$

Major or clinically-relevant non-major bleed

$9(11.3)$

Thromboembolism

$6(7.5)$

Minor bleeding

$5(6.3)$

Renal insufficiency

$3(1.1)$

Patient's wish

$3(1.1)$

other

$7(8.8)$

Alternative choice anticoagulant

Vitamin-K-Antagonist

Dabigatran

Rivaroxaban

Apixaban

$18(22.5)$

LMWH (long-term)

$5(6.3)$

None

$17(21.3)$ frequency in this cohort is nonetheless surprising and may warrant more awareness among physician who treat patients with anticoagulation.

We further investigated persistence on anticoagulation treatment in this registry and found that 80 patients (29.7\%) discontinued their baseline anticoagulation over the course of the follow-up, which translates to a discontinuation rate of 36.9 per 100 patient-years. The majority, 69 of them, switched to a different anticoagulant agent, but 11 patients discontinued anticoagulation treatment altogether. We found that after 6 months the drug persistence on the baseline anticoagulant agent was $76.7 \%$ and after 12 months further reduced to $54.7 \%$. The overall persistence in the registry is lower than in some previous reports. While in the pre-DOAC era, discontinuation frequency had been 26-28\% [17, 18], Beyer-Westendorf et al. reported a persistence rate of $81.5 \%$ in patients on treatment with rivaroxaban after a median treatment time of 544 days [19]. The most frequent reason for discontinuation of anticoagulation treatment in our registry was permanent return to sinus rhythm. This group of patients is perceived as a low-risk group and especially after successful ablation treatment, anticoagulation treatment is oftentimes discontinued in clinical practice [20]. There is, however, no evidence supporting a discontinuation of anticoagulation in patients reporting a permanent return to sinus rhythm, because AF may be asymptomatic in nature.

Moreover, we identified novel patient characteristics that decrease anticoagulation persistence. A history of bleeding, although not a risk factor for occurrence of major or CRNM bleeding was a strong risk factor for discontinuation and the composite of all bleeding events was the second most frequent reason for anticoagulation discontinuation. Further, patients with diabetes and increased leucocyte count had increased risk of discontinuation. These patients had to discontinue their baseline anticoagulation treatment predominately for emerging chronic medical issues, such as deterioration of kidney function, coronary heart and peripheral artery disease, and for safety considerations because of initiation of antiplatelet medication.

Although our registry was not intended for a direct comparison of the persistence between different anticoagulants, our data allow a cautious analysis of drug persistence on VKA and on DOACs. Patients receiving DOACs were younger, had a higher frequency of previous bleeding and had more frequently a recent diagnosis of AF. Nevertheless, patients receiving DOACs did not have inferior drug persistence compared to patients receiving VKA. In studies in German and UK primary care settings, patients on treatment with DOACs even had better drug persistence than patients on VKA [21, 22]. The benefit of DOACs over VKA concerning persistence 
may have been attenuated in the hospital setting, because treating physicians were more cautious in the use of DOACs when new medical complications emerged.

This present investigation was not without limitations. Patients were not randomized to anticoagulation treatment, which may have led to a selection bias in the distribution of comorbidities between DOAC patients and VKA patients. For example, those patients who remained on VKA treatment during the observation time were likely long-time VKA users with a stable management of the internationalnormalized ratio (INR) and personal positive experience with VKA use. The study was not suitable for assessing time in therapeutic range because INR values in between follow-up visits were not captured. Analysis of safety or efficacy between drug classes were also not permissible because there was no randomization to anticoagulation treatment. Patients were referred to the outpatient clinic for the purposes of study inclusion and not based on any selection criteria such as high risk of bleeding or stroke. There may however be an inherent selection bias in the small sample size. The low sample size further led to wide confidence interval in the logistic regression analysis. Members of the registry staff did not actively prescribe medications or suggest drug discontinuation, nor did they influence the choice of DOAC. However, in cases of adverse events, patients were encouraged to seek the advice of a physician who is independent from the registry. We were unable to analyze if drug persistence was a risk factor for occurrence of thromboembolic outcomes, because the follow-up was too short to allow statistical inference. Low adherence to anticoagulant prescription is potentially associated with increased risk of thromboembolism, but is notoriously difficult to capture and was not assessed in our investigation. There was no independent adjudication of study outcomes, but we validated outcomes internally according to criteria stated in the methods section. However, the data quality was ensured by performing all patient interviews in person with overall very good patient compliance to study procedures and a low dropout rate $(4.8 \%)$.

\section{Conclusion}

Patients with AF in this hospital setting had increased risk of thromboembolism and bleeding, which was mediated by the presence of comorbid conditions, especially a history of prior cardiovascular or cerebrovascular diseases. Further, anticoagulation treatment was frequently discontinued due to bleeding or thromboembolic complications, but also due to reasons such as permanent return to sinus rhythm, patient opinion or physician's advice. The risk of thromboembolism in the group of hospital-patients should not be underestimated and patients should be encouraged to utilize follow-up visits specifically concerning anticoagulation treatment in the first year after anticoagulation initiation.

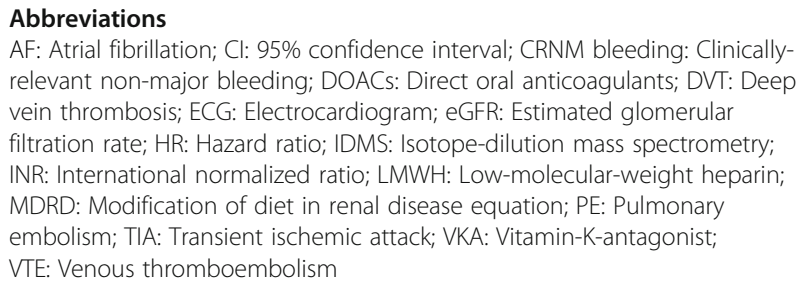

\section{Acknowledgements}

We would like to thank all clinical collaborators who contributed patients, especially Johanna Gebhart, Johannes Thaler, Julia Riedl, Giora Meron, Martin Frossard, Florian Thalhammer and Guido Gualdoni.

\section{Funding}

The study was supported by unrestricted grants from Bayer Austria and Daiichi Sankyo Austria, who did not play any role in the design of the study, the collection, analysis, or interpretation of data nor in writing the manuscript.

\section{Availability of data and materials}

The dataset supporting the conclusions of this article is available upon request.

\section{Authors' contributions}

OK conceived the study, compiled and analyzed data, and wrote the manuscript; AS interpreted data, revised the manuscript for intellectual content, and approved the manuscript for submission; HD interpreted data, revised the manuscript for intellectual content, and approved the manuscript for submission; IP interpreted data, revised the manuscript for intellectual content, and approved the manuscript for submission; CA conceived the study, analyzed data, and revised the manuscript for intellectual content and approved the manuscript for submission. All authors read and approved the final manuscript.

\section{Competing interests}

O. Königsbrügge has received honoraria for lectures from Bayer and Sanofi. A. Simon has no conflicts to disclose. H. Domanovits has given lectures for Bayer, Boehringer-Ingelheim, and Daiichi-Sankyo. I. Pabinger has received honoraria from Bayer, Daiichi-Sankyo and Boehringer-Ingelheim for occasional lectures and advisory board meetings. C. Ay has received honoraria for lectures and advisory board meetings from Bayer, Daiichi-Sankyo, Pfizer, Bristol-Myers Squibb, Boehringer-Ingelheim and Sanofi.

\section{Consent for publication}

Not applicable.

\section{Ethics approval and consent to participate}

This study was approved by the ethics committee of the Medical University of Vienna. All participants provided signed, informed consent.

\section{Author details}

'Department of Medicine I, Clinical Division of Hematology \& Hemostaseology, Medical University of Vienna, Währinger Gürtel 18-20, A-1090 Vienna, Austria. ²Department of Emergency Medicine, Medical University of Vienna, Vienna, Austria. ${ }^{3}$ Department of Medicine, Thrombosis and Hemostasis Program, McAllister Heart Institute, University of North Carolina at Chapel Hill, Chapel Hill, NC, USA.

Received: 13 October 2016 Accepted: 7 December 2016

Published online: 09 December 2016

\section{References}

1. Hart RG, Palacio S, Pearce LA. Atrial Fibrillation, Stroke, and Acute Antithrombotic Therapy: Analysis of Randomized Clinical Trials. Stroke. 2002;33:2722-7.

2. Beyer-Westendorf J, Ebertz F, Förster K, Gelbricht V, Michalski F, Köhler C, et al. Effectiveness and safety of dabigatran therapy in daily-care patients with atrial fibrillation Results from the Dresden NOAC Registry. Thromb Haemost. 2015;113:1247-57. 
3. O'Brien EC, Kim S, Thomas L, Fonarow GC, Kowey PR, Mahaffey KW, et al. Clinical Characteristics, Oral Anticoagulation Patterns, and Outcomes of Medicaid Patients With Atrial Fibrillation: Insights From the Outcomes Registry for Better Informed Treatment of Atrial Fibrillation (ORBIT-AF I) Registry. J Am Heart Assoc. 2016;5:0002721.

4. Yao X, Abraham NS, Alexander GC, Crown W, Montori VM, Sangaralingham $L R$, et al. Effect of Adherence to Oral Anticoagulants on Risk of Stroke and Major Bleeding Among Patients With Atrial Fibrillation. J Am Heart Assoc. 2016;5:1-12.

5. Palomäki A, Mustonen P, Hartikainen JEK, Nuotio I, Kiviniemi T, Ylitalo A, et al. Underuse of anticoagulation in stroke patients with atrial fibrillation - the FibStroke Study. Eur J Neurol. 2016;23:133-9.

6. Connolly SJ, Ezekowitz MD, Yusuf S, Eikelboom J, Oldgren J, Parekh A, et al. Dabigatran versus warfarin in patients with atrial fibrillation. N Engl J Med. 2009:361:1139-51.

7. Patel M, Mahaffey K, Garg J, Pan G, Singer DE, Hacke W, et al. Rivaroxaban versus warfarin in nonvalvular atrial fibrillation. N Engl J Med. 2011;365:883-91.

8. Granger CB, Alexander JH, McMurray JJV, Lopes RD, Hylek EM, Hanna M, et al. Apixaban versus warfarin in patients with atrial fibrillation. N Engl J Med. 2011;365:981-92.

9. Giugliano RP, Ruff CT, Braunwald E, Murphy SA, Wiviott SD, Halperin JL, et al. Edoxaban versus warfarin in patients with atrial fibrillation. N Engl J Med. 2013;369:2093-104.

10. Schulman S, Kearon C. Definition of major bleeding in clinical investigations of antihemostatic medicinal products in non-surgical patients. J Thromb Haemost. 2005:3:692-4.

11. Kaatz S, Ahmad D, Spyropoulos AC, Schulman S. Definition of clinically relevant non-major bleeding in studies of anticoagulants in atrial fibrillation and venous thromboembolic disease in non-surgical patients: Communication from the SSC of the ISTH. J Thromb Haemost. 2015;13:2119-26.

12. Cramer JA, Roy A, Burrell A, Fairchild CJ, Fuldeore MJ, Ollendorf DA, et al. Medication compliance and persistence: Terminology and definitions. Value Heal. 2008:11:44-7.

13. Graham DJ, Reichman ME, Wernecke M, Zhang R, Southworth MR, Levenson M, et al. Cardiovascular, Bleeding, and Mortality Risks in Elderly Medicare Patients Treated With Dabigatran or Warfarin for Nonvalvular Atrial Fibrillation. Circulation. 2015:131:157-64.

14. Korenstra J, Wijtvliet EPJ, Veeger NJGM, Geluk CA, Bartels GL, Posma JL, et al. Effectiveness and safety of dabigatran versus acenocoumarol in "real-world" patients with atrial fibrillation. Europace. 2016;18:1319-27.

15. Hecker J, Marten S, Keller L, Helmert S, Michalski F, Werth S, et al. Coagulation and Fibrinolysis Effectiveness and safety of rivaroxaban therapy in daily-care patients with atrial fibrillation Results from the Dresden NOAC Registry. Thromb Haemost. 2016;1155:1-11.

16. Bassand J-P, Accetta G, Camm AJ, Cools F, Fitzmaurice DA, Fox KAA, et al. Two-year outcomes of patients with newly diagnosed atrial fibrillation: results from GARFIELD-AF. Eur Heart J. 2016;37:2882-89.

17. Fang MC, Go AS, Chang Y, Borowsky LH, Pomernacki NK, Udaltsova N, et al. Warfarin discontinuation after starting warfarin for atrial fibrillation. Circ Cardiovasc Qual Outcomes. 2010;3:624-31.

18. Hylek EM, Evans-Molina C, Shea C, Henault LE, Regan S. Major hemorrhage and tolerability of warfarin in the first year of therapy among elderly patients with atrial fibrillation. Circulation. 2007;115:2689-96.

19. Beyer-Westendorf J, Förster K, Ebertz F, Gelbricht V, Schreier T, Göbelt M, et al. Drug persistence with rivaroxaban therapy in atrial fibrillation patients Results from the Dresden non-interventional oral anticoagulation registry. Europace. 2015;17:530-8.

20. Nührich JM, Kuck KH, Andresen D, Steven D, Spitzer SG, Hoffmann E, et al. Oral anticoagulation is frequently discontinued after ablation of paroxysmal atrial fibrillation despite previous stroke: data from the German Ablation Registry. Clin Res Cardiol. 2015:104:463-70.

21. Beyer-Westendorf J, Ehlken B, Evers T. Real-world persistence and adherence to oral anticoagulation for stroke risk reduction in patients with atrial fibrillation. Europace. 2016;18:1150-7.

22. Martinez C, Katholing A, Wallenhorst C, Freedman SB. Therapy persistence in newly diagnosed non-valvular atrial fibrillation treated with warfarin or NOAC: A cohort study. Thromb Haemost. 2016;115:31-9. 